Research Article

\title{
Conflicting Aspects in the Flat Plate Boundary Layer Conventional Solution
}

\author{
Emerson Freitas Jaguaribe \\ Departamento de Engenharia Mecânica, Centro de Tecnologia, Campus I da UFPB, João Pessoa, PB 50051-900, Brazil \\ Correspondence should be addressed to Emerson Freitas Jaguaribe; emersonjaguaribe@yahoo.com.br
}

Received 5 May 2020; Revised 19 August 2020; Accepted 15 September 2020; Published 30 September 2020

Academic Editor: Vincenzo Vespri

Copyright (C) 2020 Emerson Freitas Jaguaribe. This is an open access article distributed under the Creative Commons Attribution License, which permits unrestricted use, distribution, and reproduction in any medium, provided the original work is properly cited.

\begin{abstract}
The solution of the steady-state isothermal gas flow with zero-pressure gradient over a flat plate is classical and was presented by Blasius in 1908. Despite being reduced to a third-order Ordinary Differential Equation, nobody has solved it using just three boundary conditions. In consequence, the "solutions" (exact or numerical) are not unique, do not satisfy Prandtl's boundary layer concept, and give rise to imprecise criteria of the boundary layer thickness definition $\left(0.99 . U_{\infty}\right.$, where $U_{\infty}$ is the free stream velocity) and to idealizations such as the displacement thickness, $\delta^{*}$, and the momentum thickness, $\delta_{I}$. Even though $\eta$, the similarity parameter, is defined as a function of $x$, it is surprising that it is seen as a constant, $\eta_{\infty}$, at the limit of the boundary layer, being valid for the entire plate. It is shown that uncountable "solutions" satisfying the classical equation and its three natural boundary layer conditions can be built. The reduction technique of the Boundary Value Problem, BVP, to an Initial Value Problem, IVP, and the Perturbation Analysis Technique, used as an attempt to incorporate a fourth boundary condition to the differential equation, are discussed. A more general and direct method to deduce the classical Blasius's flat plate equation is considered, as one of the steps to rectify its solution, and shed light on the origin of the conflicting issues involved.
\end{abstract}

\section{Introduction}

In 1908, Blasius [1] presented a solution for simultaneous equations of motion and continuity on a flat plate. This system is a simplification of Prandtl's boundary layer equations characterized by the admission of incompressible flow, the steady-state regime, the isothermal gas flow, and the zero-pressure gradient all along the boundary layer. Conventionally, solving the resultant system of partial differential equations involves the use of the stream function, which allows the original differential partial equation to be transformed into a third-order nonlinear ordinary differential equation, ODE, expressed in

$$
f(\eta) \frac{\partial^{2} f(\eta)}{\partial \eta^{2}}+2 \frac{\partial^{3} f(\eta)}{\partial \eta^{3}}=0
$$

with "natural" boundary conditions:

$$
\begin{gathered}
f(0)=0, \\
\left(\frac{\partial f}{\partial \eta}\right)_{0}=0, \\
\left(\frac{\partial f}{\partial \eta}\right)_{\eta_{\infty}}=1,
\end{gathered}
$$

where $\eta$ is the similarity parameter and $f(\eta)$ the dimensionless stream function.

Although not in a realistic way, equation (1) is solved, without exception, with four boundary conditions in the specialized literature. This fact is bizarre because it is incompatible with the ODE rules and discussed in all books for the undergraduate level that deal with ODE, see, for example, Cebeci and Bradshaw [2]. In fact, a survey carried out in the literature that deals with solutions of equation (1) reveals the existence of different methods to incorporate a 
fourth boundary condition to this equation. Together with this fact, there is also misinterpretation of the generated results and the false idea that these solutions are in accordance with Prandtl's concept of the boundary layer, even when the result contradicts the formulated hypothesis. Of course, at first glance, one admits that equation (1) should be solved with just three boundary conditions. Schlichting [3], for example, p. 127, explains "the resulting differential equation is nonlinear and of third order. The three boundary conditions (7.29) are, therefore, sufficient to determine the solution completely." It is interesting to recall that Prandtl [4] commenting on the solution of equation (1) says that Blasius's solution, known as an exact solution for the flat plate boundary layer, was in fact solved by approximation, $\mathrm{p}$. 87 in Prandtl [4]. He explains the used solution mechanism; thus, "we may first ask for a simple solution. Of these we find only one, which corresponds to the potential flow; it can, however, be useful and is

$$
f=\eta+\text { constant.” }
$$

He also argued that the power series $f$ should be asymptotic, having all its coefficients a formation law. He shows that $f$ may be built from the approximation given by equation (5), where the constant is noted by $-\beta$, i.e., a negative number, which gives $f_{1}=\eta-\beta$ and as a second approximation, $f=f_{1}+f_{2}$, yielding

$$
f=\eta-\beta+\gamma \int_{\infty}^{\eta} \int_{\infty}^{\eta} e^{-(1 / 4)(\eta-\beta)^{2}} \mathrm{~d} \eta
$$

Equation (6) copied verbatim from the original.

Equation (6) gives, thus, the searched asymptotic formula. Prandtl [4] complements by saying that the resulting approximation may "be refined still further putting $f=f_{1}+f_{2}+f_{3}$, where $f_{3}$ is to be small compared to $f_{2}$, and proceeding as before." However, also according to Prandtl, this new approach is not required "because the power series converges comparatively rapidly." Blasius, in fact, used just $f_{1}$ and $f_{2}$, considering that the values of $f$, and of its first and second derivatives, $f^{\prime}$ and $f^{\prime \prime}$, are the same for the two solutions, giving the condition to determine, algebraically, $\alpha$, $\beta$, and $\gamma$, (integration constants, where " $\alpha$ occurs in the power series developed at $\eta=0 \ldots$ and $\beta$ and $\gamma$ appear in the asymptotic solution"). At the end, Prandtl [4] states "the solution of this problem is not in practice very convenient. It leads to the following values, $\alpha=0.332, \beta=1.73, \gamma=0.231$. The above calculations are due to Blasius [1]." Guran and Gwinner [5], in a 2012 publication, comment on this solution, say on p. 335, "the problem was first solved by Blasius using a series expansions method. But the proposed analytic series solution does not converge at all".

On the contrary, the $y$-direction component velocity, $v$, in the flat plate boundary layer, which results from the continuity equation in the differential form, deduced by Prandtl is given by

$$
v=-\frac{\partial \psi}{\partial x}=\frac{1}{2} \sqrt{\frac{\nu U_{\infty}}{x}}\left(\eta \frac{\partial f}{\partial \eta}-f\right) .
$$

Then, admitting in equation (7), the condition settled by equation (4), the only way to match the potential flow approach, considering equation (7) free from error, is that

$$
f\left(\eta_{\infty}\right)=\eta_{\infty}
$$

Comparison between equations (5) and (8) forces $\beta$ in equation (5) to become null, instead of being a negative number as affirmed by Prandtl [4]. On the contrary, $v$ at any $\eta_{\infty}(x)$ must be zero, i.e., all along the $x$-coordinate. However, we have to be aware that no one has used nor referred to equation (7) as a boundary condition, although, apparently, it could be chosen as another natural condition at $\eta_{\infty}$. What is the problem if equation (7) is not admitted as a boundary condition? The fact is that when $\mathrm{v}$ is considered null at $\eta_{\infty}$ to induce the existence of potential flow, it should cause some $x$-velocity components inside the boundary layer to exceed in magnitude the free stream velocity $U_{\infty}$ that is in more than 43\%, as discussed in Jaguaribe [6] and shown later in this paper. And, if $\mathrm{v}>0$ at $\eta_{\infty}$, this means there will be a flow crossing the boundary layer to counteract the effect of the fluid deceleration caused by the fluid viscosity. In other words, outside the boundary layer there will not be potential flow. Rohsenow and Choi [7], page 39, even though apparently using equation (7) to show normal parabolic profiles for the $x$-direction velocity components, $u$, do not admit the nonexistence of the potential flow, justifying the mathematical result in the exact solution of Blasius equation as "an anomaly." In spite of this, these authors, as well as Sissom and Pitts [8], emphasize that the solution of equation (1), as has been posed, is in agreement with the classical boundary layer theory.

There are many other discrepancies in the analysis of the classical Blasius's solution theory whose results, supposedly unquestionable, are impossible to give support or to correspond to some mathematical rules or existing physical principles in Fluid Mechanics, as will be shown later.

A more general and direct method to deduce the classical Blasius's flat plate equation will be presented, as one of the steps towards correctly solving Blasius equation, and bringing some explanation for the origin of these conflicting results.

\section{The Fourth Boundary Condition}

Equation (1) being a third-order nonlinear ordinary differential equation, corresponding to a two-point boundary value problem, should require just three boundary conditions. Thus, an attempt to introduce an additional boundary condition should result in a mathematical error. However, without fail, for solving the Blasius equation, either numerically or analytically, an extra boundary condition has always been used, such as

$$
\left(\frac{\partial^{2} f(\eta)}{\partial \eta^{2}}\right)_{0}=c
$$

which, with the help of a heuristic trial-and-error method, unveils the "solution." On the contrary, the common view of Blasius's solution is similar to the one by Criminale [9]: "no 
known analytical solutions exist and therefore the mean profile must be determined numerically," or to that by Parveen [10]. "The nonlinear mathematical model of the problem prohibits the use of the analytical methods." This belief has brought about through decades a great number of different methods to solve the centennial problem. Among them a mathematical technique of reducing the Boundary Value Problem, BVP_-formed by equations (1) to (3) - to an Initial Value Problem, IVP. Such a method, as mentioned by Weill [11] and by Fazio [12], was first used by Topfer [13] and seems to have dramatically influenced mathematicians and engineers since the 18th century as a means of solving a nonlinear ordinary differential equation. Indeed, as commented by Compte [14], the method applies to linear and nonlinear problems. In some circumstances, it is easy to apply, and when it does converge, it is generally the most efficient method among others.

For solving Blasius equation, however, it becomes a hard task since it requires that variables be transformed before the application of the Euler method, see Fazio [15]. To carry out this transformation one needs to admit a function, such as $F(C, \eta)$, where $C$ is an arbitrary constant to be determined in terms of $\left[\lim _{\eta \longrightarrow \infty} F^{\prime}(\eta)\right]^{-(1 / 2)}$, for a large value of $\eta$. The goal is to evaluate $f^{\prime \prime}(0)$. To do this the initial value problem for $F(\eta)$, given by equation (1) is numerically evaluated with the initial conditions $F(0)=F^{\prime}(0)=0$ and $F^{\prime \prime}(0)=1$. From this point, Rubel [16] and Fazio [17] indicate that the request to evaluate $(\mathrm{d} f / \mathrm{d} \eta)(\infty)$ cannot be fulfilled. Therefore, an approximation is required, i.e., a suitable truncate boundary layer $\eta_{\infty}$ to be used instead of $\infty$, where $\eta_{\infty}$ is large enough to guarantee also that $f^{\prime \prime}\left(\eta_{\varepsilon}\right)=\varepsilon$, where $0 \leq \varepsilon \leq 1$ and $\varepsilon \longrightarrow 0$ as $\eta_{\varepsilon} \longrightarrow \eta_{\infty}$, Fazio, 2008 [17]. Thus, the three boundary conditions are replaced by the asymptotic conditions, i.e.,

$$
\begin{array}{r}
\left(\frac{\partial f}{\partial \eta}\right)_{\eta_{\varepsilon}}=1, \\
\left(\frac{\partial^{2} f(\eta)}{\partial \eta^{2}}\right)_{\eta_{\varepsilon}}=\kappa,
\end{array}
$$

where $\kappa$ is a guess value when using the Runge-Kutta method, generally, with a shooting method, cf. Parveen [10], Bataller [18], and Criminale [9]. In practical terms, $\kappa \longrightarrow 0$, as $\eta_{\varepsilon} \longrightarrow \eta_{\infty}$. In fact, the classical Blasius's solution can be found, admitting straight away $\kappa=0$, see Asaithambi [19], or for a better mathematical explanation, Brighi and Hoernel [20]. We should be aware, however, that the technique of reducing BVP to IVP cannot always be employed. As pointed out by $\mathrm{Na}$ [21], the boundary conditions at the initial point have to be homogeneous for this transformation method to be applied. Klamkin [22], also citing $\mathrm{Na}$ [21], comments that this condition is not always necessary, but he adds that, for all BVP's and IVP's to be considered, the existence and the uniqueness of the latter should be tacitly assumed, which in turn will have implications for the former. In general, though, this is not true, see Zeldovich and Myshkis [23], pp. 32 and 33, or Nickel [24] who affirm that the boundary conditions $\left(u=\mathrm{v}=0\right.$ on $y=0$, and $u \longrightarrow \mathrm{U}_{\infty}$ as $y \longrightarrow \infty)$ ensure the existence of a solution with no guarantee, however, of uniqueness. In reality, no matter the technique to solve the classical Blasius's problem, the "solution" will not be unique if $\kappa$ in equation (11) is admitted equal to zero. Such a stratagem, or any other considering $f^{\prime \prime}\left(\eta_{\varepsilon}\right)=\varepsilon$, where $0 \leq \varepsilon \leq 1$ and $\varepsilon \longrightarrow 0$ as $\eta_{\varepsilon} \longrightarrow \eta_{\infty}$, yields just an unique value for $\alpha=\operatorname{atan}\left(f^{\prime \prime}(0)\right)$, independent of its position on the plate and there will also be a unique value for $\eta_{\infty}$. Furthermore, not only will $\left(\partial^{3} f(\eta) / \partial \eta^{3}\right)_{\eta_{\infty}}$ become null, but all derivatives of order higher than two as well, see equation (1), thus consolidating the existence of multiple "solutions" for the classical Blasius equation. These solutions recognize equation (7) as correct, i.e., in conformity with the physical reality, without accepting the nullity of the $y$-direction component velocity, $\mathrm{v}$, at $\eta_{\infty}$. This fact is another example of incorrectness in this theory. In addition, discarding the possibility of $f\left(\eta_{\infty}\right)$ to be equal to $\eta_{\infty}$ implies that $\mathrm{v}$ is not null, but constant. This can be demonstrated by deriving equation (7) in terms of $\eta$, at $\eta=\eta_{\infty}$ :

$$
\left(\frac{\mathrm{dv}}{\mathrm{d} \eta}\right)_{\eta_{\infty}}=\frac{1}{2} \sqrt{\frac{\mathrm{v} U_{\infty}}{x}}\left(\eta_{\infty}\left[\frac{\mathrm{d}^{2} f(\eta)}{\mathrm{d} \eta^{2}}\right]_{\eta_{\infty}}+\left[\frac{\partial f}{\partial \eta}\right]_{\eta_{\infty}}-\left[\frac{\partial f}{\partial \eta}\right]_{\eta_{\infty}}\right)=0 .
$$

Concluding that $\mathrm{v}$ at $\eta_{\infty}$ is a constant, which prevents the possibility of the existence of potential flow, and does not fulfill Prandtl's expectations of the boundary layer concept.

As well known, all analytical or numerical solutions found in the technical literature, reproduce the results originally presented by Blasius, see, for example, Schlichting [2], Ahmad and Al-Barakati [25], Asaithambi [19], Guran and Gwinner [5], Motsa et al. [26], Brighi and Hoernel [27], Houghton et al. [28], Katopodes [29], and Hesselgreaves et al. [30], all of them with the same particularity: not allowing the existence of the potential flow, external to the boundary layer. The fact remains that, independent of the traditional method used, it is impossible to guarantee the uniqueness of the classical Blasius's solution when a complementary condition, such as $f^{\prime \prime}\left(\eta_{\varepsilon}\right)=\varepsilon$, where $\varepsilon \longrightarrow 0$ when $\eta_{\varepsilon} \longrightarrow \eta_{\infty}$ as for example, in using the Perturbation Analysis Technique. This technique was also employed to solve Blasius equation, Van Dyke [31]. In discussing the solution of equation (1) "free of factors of 2," and using $f 1$ instead of $f^{\prime \prime}$ Van Dyke [32], comments "This is a two-point boundary-value problem, which would ordinarily require repeated guessing of $f_{1}^{\prime \prime}(0)$ to satisfy condition (7.21c) for large $\eta$." As we have pointed out above, this corresponds to adding a supplementary constraint (a "fourth boundary condition") without any guarantee of uniqueness. In fact, Van Dyke [32], in section 7.6, Uniqueness of the Blasius Solution, comes to this conclusion: "the above solution of problem (7.17) is not unique from a mathematical viewpoint." And we can add that it also prevents the nullity of the $y$-direction component velocity, $\mathrm{v}$, at $\eta_{\infty}$, setting up just a unique value of $\eta_{\infty}$ for the entire flat plate, and also giving rise to the displacement effect. Van Dyke [33] p. 2 justifies the existence of the displacement effect in flat shapes as being a result of the change of the outer tangential speed induced 
by the boundary layer itself. The same view is shared by Kaplun [34], or by Kaplun [35], p. 111: "the flow field is divided into two separate regions, that is, a boundary layer region where the flow field is obtained from boundary layer equations, and an outer region where the Euler equations are used to obtain an external flow, corrected for the displacement effect." This explanation does not hold because, as we know, all the difficulty originates in the way equation (7) was generated, causing an unbalanced mass computation in the chosen elemental differential volume.

Rohsenow and Choi [7] and Sissom and Pitts [8], in mentioning the three required conventional boundary conditions, go on emphasizing that the solution of equation (1), as has been posed, is in agreement with the classical boundary layer theory. However, an inconsistency seems to have called Rohsenow and Choi [7]'s attention, making them affirm on p. 39: "note an anomaly in this solution-in that, $\mathrm{v}$, does not go to zero at large values of $y$."

It is understandable that the flat plate boundary layer, treated as it has been, can only raise different questions about both its formulation and solution, chiefly among applied mathematicians. Weinan [36], for example, although believing that the boundary layer theory developed by Prandtl had become a standard tool, mentions, page 208, that "at the mathematical level, there is still a lack of fundamental understanding."

\section{A More General Method to Deduce the Series Solution of Equation (1)}

As already mentioned, the following partial differential equation is the classical flat plate boundary layer expression:

$$
u \frac{\partial u}{\partial x}+\mathrm{v} \frac{\partial u}{\partial y}=v \frac{\partial^{2} u}{\partial y^{2}}
$$

That was solved by Blasius [1] in conjunction with the continuity equation:

$$
\frac{\partial u}{\partial x}+\frac{\partial \mathrm{v}}{\partial y}=0
$$

and subjected to the following boundary conditions:

$$
\begin{aligned}
& u=\mathrm{v}=0 \text { at } y=0, \\
& u=\mathrm{U}_{\infty} \text { at } y \longrightarrow \infty,
\end{aligned}
$$

where $x$ and $y$ are Cartesian coordinates; $u$ is the $x$-direction component velocity; $v$ is the $y$-direction component velocity; and $\mathrm{v}$ is the fluid kinematic viscosity.

This system of equation will be solved here by means of more general expressions, which can, optionally, offer the condition to recover Blasius equation, just by particularizing values of some incorporated parameters.

Let us consider a function $\eta$ of $x$ and $y$, i.e., $\eta=\eta(x, y)$ such as

$$
\eta(x, y)=y x^{-p} A,
$$

where $p$ and $A=A\left(\mathrm{U}_{\infty}, \nu\right)$ are constants and $\mathrm{U}_{\infty}$ being the free stream velocity. If $A$ is chosen as

$$
A\left(\nu, U_{\infty}\right)=\left(\frac{U_{\infty}}{v}\right)^{q}
$$

$q$, being another constant, equation (16) turns into

$$
\eta\left(x, y, \operatorname{Re}_{x}\right)=\frac{y}{x} x^{-p-q+1} \operatorname{Re}_{x}^{q},
$$

where $\operatorname{Re}_{x}$ is the Reynolds number defined as $\operatorname{Re}_{x}=\left(U_{\infty} x / \nu\right)$.

Then, by dimensional analysis,

$$
\begin{aligned}
p+q & =1, \\
\eta\left(x, y, \operatorname{Re}_{x}\right) & =\frac{y}{x} \operatorname{Re}_{x}^{q} .
\end{aligned}
$$

Thus,

$$
\frac{\delta(x)}{x}=\eta_{\infty}(x) \operatorname{Re}_{x}^{p-1},
$$

where $\eta \longrightarrow \eta_{\infty}$ when $y \longrightarrow \infty$, i.e., $y \longrightarrow \delta$.

On the contrary, from equation (20), we have

$$
\frac{\eta}{y}=\frac{\operatorname{Re}_{x}^{q}}{x} .
$$

Let us define a function $z(\eta)$, where the first derivative, in respect to $\eta$, is given by

$$
z_{\eta}(\eta)=\frac{u}{\mathrm{U}_{\infty}}
$$

Consequently, from equations (23), (20), and (22), we have

$$
\begin{aligned}
\frac{\partial u}{\partial y} & =\mathrm{U}_{\infty} \frac{\operatorname{Re}_{x}^{q}}{x} z_{\eta \eta}, \\
\frac{\partial^{2} u}{\partial y^{2}} & =\mathrm{U}_{\infty}\left(\frac{\eta}{y}\right)^{2} z_{\eta \eta \eta}, \\
\frac{\partial u}{\partial x} & =-\mathrm{U}_{\infty} p \frac{\eta}{x} z_{\eta \eta},
\end{aligned}
$$

where $z_{\eta}, z_{\eta \eta}$, and $z_{\eta \eta \eta}$ are, respectively, the shorthand notation for the three first derivatives of $z$ in terms of $\eta$, where $p>0$. From equations (20) and (18), we can write

$$
\begin{aligned}
& \mathrm{d} y=\frac{y}{\eta} \mathrm{d} \eta, \\
& \mathrm{d} x=-\frac{x}{\eta p} \mathrm{~d} \eta,
\end{aligned}
$$

where as from equations (26) and (13), the result is

$$
\frac{\partial \mathrm{v}}{\partial \eta}=p U_{\infty} \frac{\eta}{x} z_{\eta \eta} \operatorname{Re}_{x}^{-q} .
$$

Equation (28) integrated by parts between limits 0 and $\eta$ gives,see equation (7):

$$
\mathrm{v}\left(\eta, \operatorname{Re}_{x}\right)=U_{\infty} \cdot p \cdot \operatorname{Re}_{x}^{-q}\left[\eta \cdot z_{\eta}(\eta)-z(\eta)\right] .
$$


Replacing equation (13), Blasius's partial differential equation, with equations (23) to (25) and (29) and taking into account equation (19), after some mathematical manipulations, we have

$$
p \operatorname{Re}_{x}^{2 p-1} z \cdot z_{\eta \eta}+z_{\eta \eta \eta}=0 .
$$

The "convenient" choice of $p=q=1 / 2$, see equation (19), simplifies the last equation, resulting in

$$
z . z_{\eta \eta}+2 . z_{\eta \eta \eta}=0
$$

which is the classical Blasius's flat plate equation. At the same time, equation (29) becomes

$$
\mathrm{v}\left(\eta, \operatorname{Re}_{x}\right)=\mathrm{U}_{\infty} \cdot \frac{\operatorname{Re}_{x}^{(-1 / 2)}}{2}\left[\eta \cdot z_{\eta}(\eta)-z(\eta)\right],
$$

which is the same equation (7), when $z$ is replaced by $f$.

3.1. Solving the Ordinary Differential Equation (31). The classical way of solving equation (31) requires the expansion of function $z$, and its derivatives into a Maclaurin series, which means that

$$
\begin{aligned}
z(\eta) & =\sum_{k=0}^{N} a_{k} \eta^{k}, \\
z_{\eta}(\eta) & =\sum_{k=1}^{N} k a_{k} \eta^{k-1}, \\
z_{\eta \eta}(\eta) & =\sum_{k=2}^{N} k(k-1) a_{k} \eta^{k-2}, \\
z_{\eta \eta \eta}(\eta) & =\sum_{k=3}^{N} k(k-1)(k-2) a_{k} \eta^{k-3},
\end{aligned}
$$

with the three natural boundary conditions:

$$
\begin{aligned}
z_{\eta}\left(\eta_{\infty}\right) & =1, \\
z(0) & =0, \\
z_{\eta}(0) & =0 .
\end{aligned}
$$

Replacing equation (31) with equations (33), (35), and (36) and using conditions (37) to (39), we may expand $z(\eta)$ in terms of a Maclaurin series, Kreyszig [37], where only coefficients having index given by $k=3 i+2$, are not null, $i=0,1,2, \ldots$ On the other hand, considering $a_{2}=\beta / 2$, where $\beta=z_{\eta \eta}(0)$, all the other coefficients may be determined through the recurrent formula:

$$
a_{n}=\frac{\sum_{i=2}^{n-1}\left[i(i-1) a_{i} a_{n-i-1}\right]}{2 \prod_{j=n-2}^{n} j} .
$$

Therefore,

$$
\begin{gathered}
a_{5}=-\frac{\beta^{2}}{2.5 !}, \\
a_{8}=\frac{11}{4.8 !} \beta^{3}, \\
a_{11}=-\frac{375}{8.11 !} \beta^{4}, \\
a_{14}=\frac{27897}{16.14 !} \beta^{5},
\end{gathered}
$$

and so on.

3.2. Parametrization of the Series $z(\eta)$ and Its Derivatives. In terms of $\beta$ and $\eta$, the function $z(\eta)$ and its derivatives can be expressed as follows:

$$
\begin{gathered}
z(\eta)=\beta \eta^{2} \sum_{i=0}^{N} A_{i} X^{i}, \\
z_{\eta}(\eta)=\beta \eta\left\{\sum_{i=0}^{N}(3 i+2) A_{i} X^{i}\right\}, \\
z_{\eta \eta}(\eta)=\beta \sum_{i=0}^{N}(3 i+1)(3 i+2) A_{i} X^{i}, \\
z_{\eta \eta \eta}(\eta)=\frac{\beta}{\eta} \sum_{i=1}^{N} 3 i(3 i+2)(3 i+1) A_{i} X^{i},
\end{gathered}
$$

etc., where

$$
\begin{aligned}
& X=\beta \cdot\left(\eta_{\infty}\right)^{3}, \\
& A_{0}=\frac{1}{2}, \\
& A_{1}=-\frac{1}{2.5 !} \\
& A_{2}=\frac{11}{4.8 !}, \\
& A_{3}=-\frac{375}{8.11 !} \\
& A_{4}=\frac{27897}{16.14 !},
\end{aligned}
$$

and so forth.

3.2.1. The Use of the "Fourth Boundary Condition" $z_{\eta \eta}\left(\eta_{\infty}\right)=$ 0 and Its Consequences. The "fourth boundary condition" written from equation (44) is expressed as follows:

$$
\sum_{i=0}^{N}(3 i+1)(3 i+2) A_{i} X^{i}=0 .
$$

The value of $X$, a constant, is determined from equation (48). Choosing $N=467$ in equation (48) results in $X=56.04955$. On the contrary, considering the boundary 
condition, equation (37), together with equation (43), $\beta$ is calculated as another constant, which means that the tangent at $\eta=0$, for any $x$-velocity profile, is also a constant value, despite the fact that $\delta(x)$ is a variable. Thus, $X=56.0495$ implies $\beta=0.33356$. It is worth considering that the value of $\beta$ determined by distinct authors and using different procedures is situated around 0.332 . Howarth [38], for example, considers $\beta=0.33206$; for Asaithambi [18], depending on calculation accuracy, it situates between 0.342 and 0.33205733629; for Parlange et al. [39], 0.33205734 ; Parveen [10] mentioned it as equal to 0.332059 . Consequently, given equation (46), we can conclude that $\eta_{\infty}$ is another invariant, i.e., 5.51821. It is also opportune to point out that Schlichting [2], p. 132, commenting on Blasius's solution, says "the dimensionless boundary layer thickness, $\delta \sqrt{\left(U_{\infty} / \nu x\right)}$, remains constant as long as the boundary layer remains laminar," no matter the value of $x$ in the expression $\delta \sqrt{\left(U_{\infty} / v x\right)}$. As we know, the dimensionless boundary layer thickness is in reality $\eta_{\infty}$. This result, i.e., $\eta_{\infty}$ as a constant, contrasts with the fact that, in equation (20), $\eta_{\infty}$ is a function of $x$. Thus, the constancy of the dimensionless boundary layer thickness presents an incongruity, and it comes from the use of the "fourth boundary condition," $z^{\prime \prime}\left(\eta_{\infty}\right)=0$, associated with the fact that $\beta$ is a constant too, as we have just demonstrated. On the contrary, we should remember that $z^{\prime \prime}\left(\eta_{\infty}\right)=0$ implies that $z^{\prime \prime \prime}\left(\eta_{\infty}\right)=0$, as well as that $z^{(i v)}\left(\eta_{\infty}\right)=0$, and so on, see equation (31), generating different "solutions" for the Blasius equation, all of them featuring $\beta$ and $\eta_{\infty}$, as constants.

3.2.2. About Equation (30). The classical equation (30) as well as equation (7) are also, as already shown, a result of the use of the continuity equation in differential form, i.e., equation (14). Equation (14), unconditionally used in many physical situations, does not take into account the particular aspect of the uniform velocity profile (uniform flow) intrinsic to the design of Blasius's flat plate flow. Therefore, all the correlations resultant from equation (31) cannot fit into what was ideally expected by Prandtl [4], namely, the occurrence of the potential flow. In fact, as noticed by Rohsenow and Choi [7], page 39, and by Bejan [40], page 58, the solution is not consonant with the potential flow, and it is not asymptotic, as we have also demonstrated above, in Section 2. The impossibility of the classical Blasius equation solution to be in agreement with the potential flow is so evident that can be easily seen by evoking the solution in terms of the series $z(\eta)$, i.e., equation (42). To do so, we need to consider the natural conditions (37) to (39) and equation (49), required to guarantee the existence of the potential flow, coming from equation (30), i.e.,

$$
z\left(\eta_{\infty}\right)=\eta_{\infty}
$$

Thus, the association of condition equation (49), with equation (42), and of condition (37) with equation (43), gives

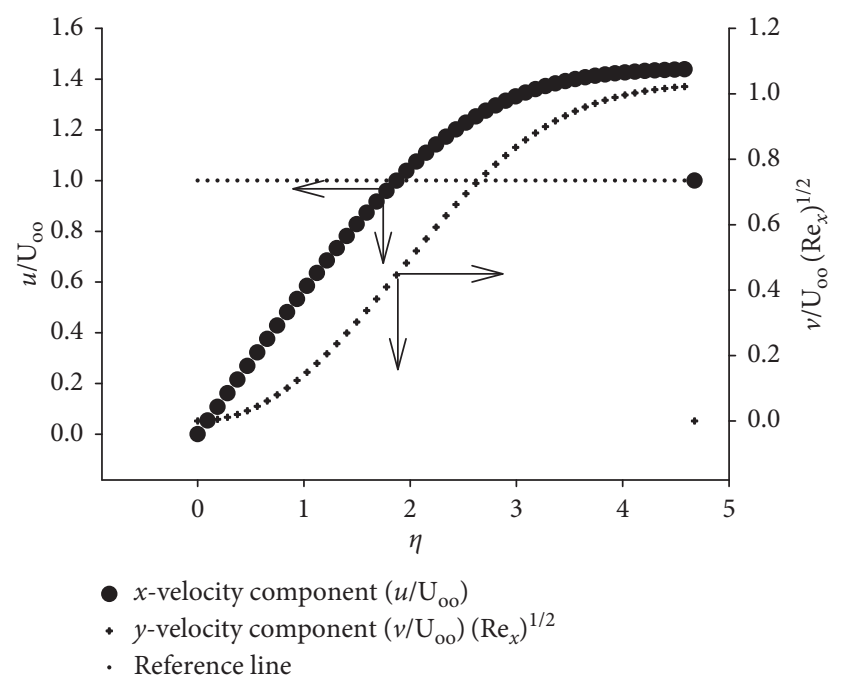

Figure 1: Dimensionless velocity profiles ( $x$ - and $y$-components) determined considering equation (7) or (31) as correct.

$$
\sum_{i=0}^{N}(3 i+1) A_{i} X^{i}=0
$$

Equation (50) allows the evaluation of $X$. On the contrary, knowing $X$, the combination of equation (43), and condition (37) results in equation (51), and, consequently, in the evaluation of parameter $P$ :

$$
P=\sum_{i=0}^{N}(3 i+2) A_{i} X^{i}=\frac{1}{\beta \eta_{\infty}} .
$$

Then, using equations (46) and (51), we determine

$$
\eta_{\infty}=\sqrt{X . P}
$$

and thus,

$$
\beta=\frac{1}{P \cdot \eta_{\infty}} .
$$

Considering in equation (50), $N=461$, we determine $X=58.81542$, and consequently $\eta_{\infty}=4.67551$ and $\beta=0.57544$, allowing the plot of Figure 1, which presents the dimensionless $x$ - and $y$-direction component velocities in terms of $\eta$. In Figure 1, we see that the dimensionless $y$ component velocity profile allows the existence of the potential flow, when all of a sudden, at $\eta_{\infty}, v$ goes to zero. As to the dimensionless $x$-direction component velocity, $u / \mathrm{U}_{\infty}$, we notice that Figure 1 shows dimensionless velocity magnitudes that overpass the constant reference line, drawn in parallel with axis- $\eta$, which crosses the ordinate axis at $u$ / $\mathrm{U}_{\infty}=1$. Such evidence contrasts with the reality and indicates that $z\left(\eta_{\infty}\right)$ cannot be, by any means, equal to $\eta_{\infty}$, and therefore, if equation (31) truly represents the $y$-direction component velocity, the potential flow will never exist, nor even the known boundary layer outline. In this respect, Rohsenow and Choi [7], page 39, show that the $y$-direction component velocity is given by 


$$
\mathrm{v}_{y}=0.865 \mathrm{U}_{\infty} \sqrt{\frac{v}{x \mathrm{U}_{\infty}}},
$$

which means that the asymptotic behavior does not exist, neither the potential flow. Furthermore, these inconsistencies cause, as expected, a problem with mass and momentum balances, requesting the introduction of two artificial parameters, that is, the displacement thickness, $\delta^{*}$, and the momentum thickness, $\delta_{I}$, in order to complement the quantities in deficit.

\section{Conclusion}

As we have seen, the classical flat plate Blasius's solution presents two kinds of inconsistency: mathematical and physical. A flagrant mathematical fault is committed when the Blasius's third-order differential equation is solved using four boundary conditions. On the contrary, it is proved that the resultant velocity profiles, do not attend to the physical definition of the boundary layer as defined by Prandtl. It may, therefore, come as something of a shock to realize that the classical image of the boundary layer of a flat plate is not reproduced under the classical mathematical model, despite the utilization of the three required standard boundary conditions. From the present discussion, it follows that the reasons for these inconsistencies are

(a) The misinterpretation of the fact that $\beta=z_{\eta \eta}(0)$ is a constant and an unknown parameter, $z$ being the solution of the third-order nonlinear ordinary differential equation, obtained by transformation from the original Blasius's partial differential equation, see equation (31). In consequence, a fourth boundary condition is required to "solve" the ODE, resulting in a "solution" that is not unique and bringing about a series of other inconsistencies: just one value of $\eta_{\infty}$ for all the entire flat plate; the need to lay hands on two artificial idealizations: the displacement thickness, $\delta^{*}$ and the momentum thickness, $\delta_{I}$; the nonexistence of any explicit relation to the local Reynolds number, $\mathrm{Re}_{\mathrm{x}}$.

(b) The use of the continuity equation in the differential form to deduce the $y$-direction component velocity, which resulted, as we have seen, in the fact that $\mathrm{v}\left(\eta_{\infty}\right)$ does not go to zero, thus, preventing the formation of the potential flow zone.

(c) The determination of the standard form of the similarity solution by means of a transformation of the variables $x$ and $y$ and the stream function $\psi$ so that the original Blasius's partial differential equation becomes independent of $x$. This implies that the dimensionless similarity variable, as $y \longrightarrow \infty$, i.e., $\eta_{\infty}$, remains invariant, in other words, not dependent on $x$, see Katopodes [29] on pages 661 and 662 .

While the main goal of this paper is to shed some light on the inaccurate and erroneous aspects involved in the Blasius's classical solution, it also brings a new methodology that by avoiding the utilization of the stream function as the way to transform variables, and it generates more general expressions for the solution of the flat plate boundary layer, under the same constraints as those used by Blasius. Taking into account equation (30), we notice that, for $p \neq 1 / 2$, the flat plate equation becomes conditioned to the values of $\mathrm{Re}_{\mathrm{x}}$. This condition turns the correlation more susceptible to produce the solution of Blasius equation in conformity with the mathematical and physical requirements and prevents the illogical deduction that results in $\eta_{\infty} \neq \eta_{\infty}(x)$.

\section{Data Availability}

The data used to support the findings of the study are available from the corresponding author upon request.

\section{Conflicts of Interest}

The author declares that he has no conflicts of interest.

\section{References}

[1] H. Blasius, "Grenzschichten in Flussigkeiten mit kleiner Reibung," NACA Technical Memorandum, vol. 1256, 1908.

[2] T. Cebeci and P. Bradshaw, Momentum Transfer in Boundary Layers, p. 407, Hemisphere Publishing Corporation, New York, NY, USA, 1977.

[3] H. Schlichting, Boundary Layer Theory, Vol. 960, Springer, Berlin, Germany, 1960.

[4] L. Prandtl, "The mechanics of viscous fluids," Aerodynamic Theory, vol. 3, pp. 155-162, 1935.

[5] A. Guran and J. Gwinner, "An enhanced numerical solution of Blasius equation by means of the method of differential quadrature," in Proceedings of the 18th international conference on Engineering Mechanics, Svratka, Czech Republic, May 2012.

[6] E. Jaguaribe, Rethinking Von Karman's Solution of the Laminar Flat Plate Boundary Layerpp. 334-339, Asian Congress of Fluid Mechanics, Singapore, 6th edition, 1995.

[7] W. M. Rohsenow and H. Y. Choi, Heat, Mass, and Momentum Transfer, Prentice-Hall, Upper Saddle River, NJ, USA, 1961.

[8] L. Sissom and D. Pitts, Elements of Transport Phenomena, McGraw-Hill Book Company, New York, NY, USA, 1972.

[9] R. D. J. W. O. Criminale and T. L. Jackson, Theory and Computation of Hydrodynamic Stability, Cambridge University Press, Cambridge, UK, 1973.

[10] S. Parveen, "Numerical solution of non-linear differential equation by using shooting techniques," International Journal of Mathematics and Its Applications, vol. 4, no. 1-A, 2016.

[11] H. Weill, "On the differential equations of the simplest boundary-layer problems," Annals of Mathematics, vol. 43, no. 2, 1942.

[12] R. Fazio, "A survey on free boundary identification of the truncated boundary in numerical BVPs on infinite intervalsfication of the truncated boundary in numerical bvps on infinite intervals," Journal of Computational and Applied Mathematics, vol. 140, no. 1-2, pp. 331-344, 2002.

[13] C. Töpfer, "Bomerkungen zu dem aufsatz von h. Blasius, "grenzschichten in flüssigkeiten mit kleiner reibung"” Zeitschrift für Angewandte Mathematik und Physik, vol. 60, pp. 397-398, 1912.

[14] S. D. Compte, Elementary Numerical Analysis an Algorithm Approach, p. 268, McGraw-Hill, New York, NY, USA, 1965. 
[15] R. Fazio, "Blasius problem and Falkner-Skan model: Töpfer's algorithm and its extension," Computers \& Fluids, vol. 73, pp. 202-209, 2013.

[16] L. A. Rubel, "An estimate of the error due to the truncated boundary in the numerical solution of the Blasius equation," Quarterly of Applied Mathematics, vol. 13, no. 2, pp. 203-206, 1955.

[17] R. Fazio, "Transformation methods for the Blasius problem and its recent variants," In Proceedings of the World Congress on Engineering, vol. 2, 2008.

[18] R. C. Bataller, "Numerical comparisons of Blasius and sakiadis flows," Matematika, vol. 26, pp. 187-196, 2010.

[19] A. Asaithambi, "Solution of the Falkner-Skan equation by recursive evaluation of Taylor coefficients," Journal of Computational and Applied Mathematics, vol. 176, no. 1, pp. 203-214, 2005.

[20] B. Brighi and J.-D. Hoernel, "On similarity solutions for boundary layer flows with prescribed heat flux flows with prescribed heat flux," Mathematical Methods in the Applied Sciences, vol. 28, no. 4, pp. 479-503, 2005.

[21] T. Y. Na, "Transforming boundary conditions to initial conditions for ordinary differential equations differential equations," SIAM Review, vol. 9, no. 2, pp. 204-210, 1967.

[22] M. S. Klamkin, "Transformation of boundary value problems into initial value problems," Journal of Mathematical Analysis and Applications, vol. 32, no. 2, pp. 308-330, 1970.

[23] I. B. Zeldovich and A. D. Myshkis, Elements of Applied Mathematics, Jeevansons Publications, Daryaganj, India, 1976.

[24] K. Nickel, "Prandtl's boundary-layer theory from the viewpoint of a mathematician," Annual Review of Fluid Mechanics, vol. 5, no. 1, pp. 405-428, 1973.

[25] F. Ahmad and W. H. Al-Barakati, "An approximate analytic solution of the Blasius problem," Communications in Nonlinear Science and Numerical Simulation, vol. 14, no. 4, pp. 1021-1024, 2009.

[26] S. P. Motsa, T. G. Marevo, P. Sibanda, and S. Shateyi, "An improved spectral homotopy analysis method for solving boundary layer problems," Boundary Value Problems, vol. 2011, 2011.

[27] B. Brighi and J.-D. Hoernel, "On a general similarity boundary layer equation," Acta Mathematica Universitatis Comenianae, vol. 77, no. 1, 2008.

[28] E. L. Houghton, P. W. Carpenter, S. Collicott, and D. Valentine, Aerodynamics for Engineering Students, Butterworth-Heinemann, Oxford, UK, 7th edition, 2016.

[29] N. D. Katopodes, Free-surface Flow: Environmental Fluid Mechanics, Butterworth-Heinemann, Oxford, UK, 2018.

[30] J. E. Hesselgreaves, R. Law, and D. Reay, Compact Heat Exchangers: Selection, Design and Operation, ButterworthHeinemann, Oxford, UK, 2016.

[31] M. Van Dyke, Perturbation Methods in Fluid Mechanics, Vol. 964, Academic Press, New York, NY, USA, 1964.

[32] M. Van Dyke, "Perturbation methods in fluid mechanics/ annotated edition," NASA STI/Recon Technical Report A, vol. 75, 1975.

[33] M. D. Van Dyke, "A survey of higher-order boundary-layer theory," SUDAAR, vol. 326, 1967.

[34] S. Kaplun and P. Lagerstrom, "Asymptotic expansions of Navier-Stokes solutions for small Reynolds numbers," Indiana University Mathematics Journal, vol. 6, no. 4, pp. 585-593, 1957.
[35] S. Kaplun, "The role of coordinate systems in boundary-layer theory," Zeitschrift für angewandte Mathematik und Physik ZAMP, vol. 5, no. 2, pp. 111-135, 1954.

[36] E. Weinan, "Boundary layer theory and the zero-viscosity limit of the Navier-Stokes equation," Acta Mathematica Sinica, English Series, vol. 16, no. 2, pp. 207-218, 2000.

[37] E. Kreyszig, Advanced Engineering Mathematics, John Wiley \& Sons, Hoboken, NJ, USA, 2007.

[38] L. Howarth and L. Bairstow, "On the solution of the laminar boundary layer equations," Proceedings of the Royal Society A: Mathematical, Physical and Engineering Sciences, vol. 164, no. 919, pp. 547-579, 1938.

[39] J. Y. Parlange, R. D. Braddock, and G. Sander, "Analytical approximations to the solution of the Blasius equation," Acta Mechanica, vol. 38, no. 1-2, pp. 119-125, 1981.

[40] A. Bejan, Convection Heat Transfer, Wiley, Hoboken, NJ, USA, 4th edition, 2003. 